\title{
Actividad física y tiempos de comida en teletrabajadores y trabajadores presenciales de una universidad estatal de Costa Rica
}

\author{
Lourdes Arce Espinoza ${ }^{1}$ (D) \& Karla Rojas Sáurez ${ }^{1}$ \\ 1. Universidad Estatal a Distancia, Vicerrectoría Ejecutiva, Servicio Médico UNED, 2050 Sabanilla, San José, Costa \\ Rica; larce@uned.ac.cr; krojas@uned.ac.cr
}

\begin{abstract}
Physical activity and meal times in teleworkers versus presential workers of a state university of Costa Rica." Introduction: Teleworking is a relatively new labor modality in Costa Rica; however, sedentary lifestyle and mealtime disorders may lead to obesity and cardiovascular disease in teleworkers. Objective: To measure if teleworkers have different levels of physical activity and meal times than their counterparts. Methods: We surveyed online 241 teleworkers and 223 presential workers. Results: Presential workers reported more physical activity and better compliance with meal times. Conclusion: Teleworkers in this institution need to change activity and eating practices to reduce the danger of obesity and cardiovascular diseases.
\end{abstract}

Keywords: quality of life, well-being, health, labor flexibility, social commitment, telework, face-to-face work.
RESUMEN. Introducción: El teletrabajo es una modalidad laboral relativamente nueva en Costa Rica; sin embargo, el estilo de vida sedentario y deficiencias en los horarios de comida pueden provocar obesidad y enfermedades cardiovasculares en los teletrabajadores. Objetivo: Medir si los teletrabajadores tienen diferentes niveles de actividad física y horarios de comida que sus contrapartes. Métodos: Encuestamos en línea a 241 teletrabajadores y 223 trabajadores presenciales. Resultados: Los trabajadores potenciales informaron más actividad física y un mejor cumplimiento de los horarios de las comidas. Conclusión: Los teletrabajadores de esta institución necesitan cambiar las prácticas de actividad y alimentación para reducir el peligro de obesidad y enfermedades cardiovasculares.

Palabras clave: calidad de vida, bienestar, salud, flexibilidad laboral, teletrabajo, trabajo presencial. 
El Teletrabajo (TT) se define como aquella actividad laboral que se realiza fuera de la oficina de la empresa y con mediación de la tecnología. Su inicio data de los años 70's, evolucionando la forma de trabajar a partir del desarrrollo de la tecnología en las últimas décadas. La incorporación paulatina de esta modalidad en las industrias tiene gran aceptación debido a los beneficios tanto para las empresas como para los trabajadores (Brindusa, Cozzolino, \& Lacuesta, 2020; Gómez, Guarín, Uribe, \& Vergel, 2020).

Existen diversas variaciones del TT, cada una se ajusta a la realidad y la necesidad del empleador, así como al perfil del trabajador. El trabajo presencial, sigue estando vigente, sin embargo, el TT se ha abierto paso en las diferentes ocupaciones, posicionándose como una innovadora modalidad laboral (Caamaño, 2010; Núñez \& Quirós-Ramírez, 2016; Sánchez, Montenegro, \& Medina, 2019).

Aunque son muchos los beneficios que se le atribuyen al $T$, también existen desventajas. Dentro de las desventajas que se mencionan son la propensión al sedentarismo y desórdenes en los horarios y tiempos de comidas, produciendo mayor riesgo de desarrollar obesidad (Walter, PérezSimon, Nagham-Ngwessitcheu, \& Vázquez-Ubago, 2014; Bidikay, 2018; Sánchez et al., 2019; Vries, Tummers, \& Vekkers, 2019).

EI TT, puede fomentar el sedentarismo y desórdenes en los tiempos de comida, debido a que la misma dinámica de esta modalidad, influye en la poca percepción del tiempo, dando con esto a que no se realicen los descansos y por ende se saltan los tiempos de comida, como las meriendas o el almuerzo o cena. Además, produce menos movimiento físico, debido a que no debe trasladarse, por lo cual, puede aumentar el sedentarismo en las personas que no son ya de por si activas ( Tapasco \& Giraldo, 2016; Luque \& Camargo, 2017; Vries et al., 2019).

Diversos estudios confirman que las personas que son poco activas y cuya una actividad física consistía en los traslados al trabajo, después de optar por la modalidad de TT, por uno, dos o más días a la semana, aumentó el nivel de sedentarismo ya que no deben trasladarse. Sumado a esto se encuentra también la mayor disponibilidad de consumo de alimentos entre comidas lo que genera un aumento de peso y desorden en los tiempos de comida (Cataño \& Gómez, 2014; Tapasco \& Giraldo, 2016; Centro Internacional para el desarrollo del Teletrabajo, 2017).

Este estudio ofrece información científica original sobre este tema en el ámbito que se realizó; los resultados pueden ser utilizados en otros estudios y en ambientes similares. El objetivo principal del estudio es medir si los teletrabajadores tienen diferentes niveles de actividad física y horarios de comida que sus contrapartes presenciales.

\section{MATERIALES Y MÉTODOS}

Instrumento: El instrumento fue diseñado para aplicarlo en línea utilizándose el programa LIMESURVEY. Consta de siete secciones: 1. Datos personales, 2. Datos de salud, 3. Nutrición, 4. Actividad física, 5. Calidad de vida, 6. Estrés, 7. Uso de equipo y seguridad. Sin embargo, para los efectos del presente estudio solo se consideran los resultados de las secciones: datos personales, actividad física y nutrición. El instrumento fue validado por juicio de expertos, se envió a 30 personas escogidas al azar para poder determinar de manera independiente la relevancia y congruencia del contenido del cuestionario con el contenido teórico, la claridad en la redacción y el sesgo o tendenciosidad en la formulación de los ítems, es decir, si sugieren o no una respuesta (HernándezSampieri, Fernández-Collado, \& Baptista, 2014). 
Procedimiento: Con respecto a los teletrabajadores, se solicitó al Programa de Teletrabajo la lista de los funcionarios teletrabajadores que tuvieran al menos un año de estar en esta modalidad de trabajo; posteriormente se envió un correo personalizado que incluía la invitación para participar en el estudio, en este correo se indicaba un enlace para acceder el cuestionario y responderlo a través de la web. Con respecto a los trabajadores presenciales, se solicitó a la Oficina de Recursos Humanos, la lista de trabajadores activos, con cuenta de correo electrónico y que al menos tuvieran un año de laborar en la institución. Se eliminaron del listado los Teletrabajadores y posteriormente se le asignó un número de la lista y con un programa en línea generador de números al azar, se determinó un listado de 350 funcionarios presenciales, a los cuales se les envió un correo personalizado que incluía la invitación para participar en el estudio. Durante el periodo en que se mantuvo activo el cuestionario se enviaron tres recordatorios a los que no lo habían contestado con el fin de motivar la participación. Se envió a 300 teletrabajadores y 350 funcionarios presenciales, lográndose obtener un $80 \%$ de respuesta en la población teletrabajadora y $64 \%$ del grupo de trabajadores presenciales; se mantuvo activo desde el 01 de octubre de 2019 al 14 de diciembre del 2019.

\section{RESULTADOS}

En relación con las características sociodemográficas y laborales de los funcionarios teletrabajadores se obtuvo que, existe mayor presencia del sexo femenino, la mayoría de los funcionarios tienen entre 31 a 40 años y más, son casados, no tienen hijos. En mayor proporción se cuenta con formación universitaria completa y cuentan con más de 11 años de antigüedad laboral. En su mayoría ocupan puestos profesionales y laboran en la Vicerrectoría Académica.

En relación con las características sociodemográficas y laborales de los funcionarios presenciales, se obtuvo que, existe mayor presencia del sexo femenino, la mayoría de los funcionarios tienen menos de 30 años, son divorciados, separados o viudos, tienen dos o más hijos. En mayor proporción se cuenta con formación universitaria completa y cuentan con menos de 10 años de antigüedad laboral. En su mayoría ocupan puestos administrativos y laboran en la Vicerrectoría Académica.

Los resultados rechazan la hipótesis alternativa de que los funcionarios teletrabajadores realizan más actividad física que los trabajadores presenciales, al verificarse una diferencia significativa con relación a la modalidad de trabajo ( $U$ de Mann-Whitney $(n 1=241, n 2=223)=21$ $452,0, p=0,001$ ); y con respecto a la hipótesis de que los funcionarios teletrabajadores realizan más tiempos de comida que los trabajadores presenciales, también se rechaza la hipótesis alternativa, ya que se comprueba una diferencia significativa entre las modalidades ( $U$ de Mann-Whitney ( $\mathrm{n} 1=$ $241, \mathrm{n} 2=223)=13027,0, p=0,000)$. Por tanto, se rechazan ambas hipótesis alternativas. 


\section{CUADRO 1}

Distribución porcentual de las características sociodemográficas y laborales de los funcionarios según modalidad de trabajo

\begin{tabular}{|c|c|c|}
\hline Característica & $\begin{array}{c}\text { Teletrabajador } \\
\text { (\%) }\end{array}$ & $\begin{array}{c}\text { Trabajador } \\
\text { presencial (\%) }\end{array}$ \\
\hline \multicolumn{3}{|l|}{ Sexo } \\
\hline Femenino & 75,0 & 54,0 \\
\hline Masculino & 25,0 & 46,0 \\
\hline \multicolumn{3}{|l|}{ Grupo de edad } \\
\hline Menos de 30 & 5,0 & 54,0 \\
\hline 31 a 40 & 42,0 & 46,0 \\
\hline 41 a 50 & 32,0 & 0,0 \\
\hline Más de 50 & 21,0 & 0,0 \\
\hline \multicolumn{3}{|l|}{ Estado civil } \\
\hline Soltero & 27,0 & 0,0 \\
\hline Casado & 48,0 & 12,0 \\
\hline Divorciado, separado, viudo & 15,0 & 60,0 \\
\hline Unión Libre & 10,0 & 28,0 \\
\hline \multicolumn{3}{|l|}{$\mathbf{N}^{\circ}$ de hijos(as) } \\
\hline Ninguno & 45,0 & 3,0 \\
\hline 1 hijo(a) & 25,0 & 33,0 \\
\hline 2 hijos(as) & 24,0 & 39,0 \\
\hline 3 o más hijos(as) & 6,0 & 25,0 \\
\hline \multicolumn{3}{|l|}{ Escolaridad } \\
\hline Universitaria completa & 95,0 & 63,0 \\
\hline Universitaria incompleta & 4,0 & 23,0 \\
\hline Otro & 1,0 & 14,0 \\
\hline \multicolumn{3}{|l|}{ Antigüedad laboral (años) } \\
\hline Menos de 10 & 42,0 & 50,0 \\
\hline 11 a 20 & 44,0 & 27,0 \\
\hline 21 a 30 & 10,0 & 14,0 \\
\hline Más de 30 & 4,0 & 9,0 \\
\hline \multicolumn{3}{|l|}{ Puesto } \\
\hline Personal profesional & 88,0 & 49,0 \\
\hline Personal administrativo & 12,0 & 51,0 \\
\hline \multicolumn{3}{|l|}{ Departamento } \\
\hline Rectoría & 12,0 & 12,0 \\
\hline Vicerrectoría Académica & 60,0 & 50,0 \\
\hline Vicerrectoría Ejecutiva & 15,0 & 30,0 \\
\hline Vicerrectoría Planificación & 9,0 & 3,0 \\
\hline Vicerrectoría Investigación & 4,0 & 5,0 \\
\hline
\end{tabular}




\section{DISCUSIÓN}

Se rechaza la hipótesis alternativa de que los funcionarios teletrabajadores realizan más actividad física que los trabajadores presenciales; lo cual puede deberse a diferencias de: sexo, edad y número de hijos.

La actividad física es definida como todos aquellos movimientos que realiza el ser humano para mejorar la capacidad motora y muscular, es producto de la contracción muscular y aumenta el gasto de energía. Tenemos entonces, que la intencionalidad de la actividad física es mantener o mejorar la composición corporal. Tenemos también que la actividad física disminuye con la edad, es decir, conforme se aumenta la edad, la frecuencia de realización de la actividad física es menor con respecto a las poblaciones más jóvenes y la intensidad también disminuye, optándose por actividades que requieran menos esfuerzo físico (Mikel, Ander, \& Urdampilleta, 2012).

Se ha encontrado que las personas menores de 30 años, realizan más ejercicio que las mayores de 30, esta disminución se debe a que la capacidad de exigencia y de rendimiento físico disminuye con los años, en algunos casos motivados también por la aparición de enfermedades, que provocan que las personas, entren en un estado de comodidad con el sedentarismo (Sierra, Escobar, \& Melo, 2016; Tavares, 2017; Ordóñez, 2018).

Estudios confirman además que las mujeres tienden a ser mas sedentarias que los hombres, puesto que por su rol laboral y familiar dedican menos tiempo a la realización de actividad física, estos mismos estudios indican que las mujeres son más sedentarias en todos los grupos de edad y las que se ejercitan lo hacen de forma más irregular que los hombres; afirman además que la inequidad de género tiende a crear una brecha entre los hombres y mujeres en todos los ámbitos incluyendo la actividad física (Castañeda-Vázquez, Zagalaz-Sánchez, Cachón-Zagalaz, \& RomeroGranados, 2014; Dosal, Mejía, \& Cardevilla, 2017; Secretaría de Salud Laboral y Secretaría de las Mujeres, 2017).

Se ha encontrado además que el tener hijos se asocia a una mayor realización de actividad física, debido a que las personas desean estar en las mejores condiciones de salud posibles para poder compartir calidad de vida con su familia (Martín, Barripedro, Martínez del Castillo, JiménezBeatty, \& Rivero-Herráiz; 2014; Universidad de Costa Rica, 2016).

Se rechaza la hipótesis alternativa de que los funcionarios teletrabajadores realizan más tiempos de comida que los funcionarios presenciales; esto puede explicarse como la característica de la disponibilidad de comida, lo cual genera un constante consumo de alimentos, conocido como "síndrome de patata en el escritorio" (Gómez et al., 2020), el cual genera obesidad y desórdenes en los tiempos de comida.

Una desventaja encontrada en el TT, es el "síndrome de patata en el escritorio", el cual hace referencia a consumo de alimentos poco nutritivos y disminución de los tiempos de comida importantes en la nutrición del ser humano. En la oficina o espacio laboral el contexto permite que se cumplan los recesos y tiempos de comida de forma puntual, mientras que en el TT ese contexto no existe, por lo cual, las personas tienden a saltarse tiempos de comida y no realizar los descansos, pues en el mismo espacio de trabajo, consumen algún alimento (Benjumea-Arias, Villa-Enciso, \& Valencia-Arias, 2016; Sierra et al., 2016; Bidikay, 2018; Álvarez, 2020).

La regulación de la autonomía en esta modalidad, es vital para mantener los horarios, los descansos y la productividad, se ha visto que, muchos teletrabajadores pasan la mayoría de la jornada, sin realizar al menos una pausa; es habitual también que consuman algún alimento en el mismo espacio de trabajo, esto debido a que el contexto de oficina está ausente y los funcionarios al no tener interrupciones se dedican de lleno al trabajo, saltándose descansos y tiempos de comida (Pérez, 2010; Alvarez, 2012; Vicente-Herrero, Torres, Torres, Ramírez, \& Capdevila, 2018). 
Si bien los beneficios del TT superan con creces al trabajo presencial, también tiene algunas desventajas que podrían afectar la salud física y por ende podrían incrementar el riesgo de sedentarismo y obesidad, propiciando el desarrollo de enfermedades cardiovasculares; (Pérez, 2010; Knox, Biddle, Esliger, Piggin, \& Sherar, 2014; Henke et al., 2016; Organización Internacional de Trabajo, 2016; Sánchez et al., 2019; Gómez et al., 2020). Sin embargo, se podría concluir también que un programa de acompañamiento al teletrabajador en estos aspectos de salud, donde se promueva el ejercicio físico y la importancia de una nutrición adecuada respetando tiempos de comida y comidas balanceadas, podría solventar estas desventajas y reducir el riesgo del sedentarismo y desórdenes en los habitos alimentarios.

\section{AGRADECIMIENTOS}

Agradecemos a Julián Monge de la Vicerrectoría de Investigación UNED, por su apoyo, guía y acertados consejos en el desarrollo de esta investigación. A Ligia Bermúdez Mesén de la Vicerrectoría de Investigación UNED, por su apoyo en esta investigación. A todos los funcionarios que completaron el instrumento.

\section{ÉTICA, CONFLICTO DE INTERESES Y DECLARACIÓN DE FINANCIAMIENTO}

Las autoras declaran haber cumplido con todos los requisitos éticos y legales pertinentes, tanto durante el estudio como en el manuscrito; que no hay conflictos de interés de ningún tipo, y que todas las fuentes financieras se detallan plena y claramente en la sección de agradecimientos. Asimismo, están de acuerdo con la versión editada final del documento. El respectivo documento legal firmado se encuentra en los archivos de la revista.

La contribución de los autores es como se detalla a continuación: L.A.E.: Elaboración del Proyecto de Investigación y participación activa durante todas las etapas de la Investigación: elaboracióndel proyecto e instrumento, sensibilización de la población, recolección de la información, tabulación, análisis estadístico y elaboracióndel artículo. K.R.S.: Supervisión y revisióndel proyecto de Investigación en todas las etapas. 


\section{REFERENCIAS}

Álvarez, C. H. (2020). Del recurso al teletrabajo como medida de emergencia al futuro del trabajo a distancia. Lan Harremanak: Revista de Relaciones Laborales, 43, 1-24.

Alvarez, A. R. (2012). La introducción del teletrabajo en la administración general del estado mejoras profesionales y personales. Sociología del Trabajo, nueva época, (75), 73-92.

Benjumea-Arias, M., Villa-Enciso, E., \& Valencia-Arias, J. (2016). Beneficios e impactos del teletrabajo en el talento humano. resultados desde una revisión de literatura. Revista CEA, 2(4), 59-73.

Bidikay, T. (2018). Teletrabajo, necesidad y solución en Méjico. Un estudio exploratorio de sus barreras culturales y obstáculos. Revista Latinoamericana de Investigación en Organizaciones Ambiente y Sociedad, 9,(12) 153-176.

Brindusa, A., Cozzolino, M., \& Lacuesta, A. (2020). El teletrabajo en España. Boletín económico: Banco de España, 18, 118.

Caamaño, R. E. (2010). El teletrabajo como una alternativa para promover y facilitar la conciliación de responsabilidades laborales y familiares. Revista de Derecho de la Pontificia Universidad Católica de Valparaíso, 35(2), 79-105.

Castañeda-Vázquez, C., Zagalaz-Sánchez, M. C.-B., Cachón-Zagalaz, J., \& Romero-Granados, R. (2014). Características de la práctica deportiva en función del género. Estudiantes de la Facultad de Ciencias de la Educación: Universidad de Sevilla. Retos. Nuevas tendencias en Educación Física, Deporte y Recreación, 25, 63-67.

Cataño, R. S., \& Gómez, R. N. (2014). El concepto de teletrabajo: aspectos para la seguridad y salud en el empleo. CES Salud Pública, 5(1), 82-91.

Centro Internacional para el desarrollo del Teletrabajo. (2017). Primer Informe sobre el estado del teletrabajo en Costa Rica. San José, Costa Rica: CIIDTT. Recuperado de: https://cidtt.org/wpcontent/uploads/2018/03/PrimerInformeTeletrabajo-2.pdf

Dosal, U. R., Mejía, C. M., \& Cardevilla, O. L. (2017). Deporte y equidad de género. Economía UNAM, 14(40), 121-133.

Gómez, S., Guarín, I., Uribe, S., \& Vergel, L. (2020). Prevención de los peligros y promoción de entornos saludables en el teletrabajo desde la perspectiva de la salud pública. Aibi revista de investigación, administración e ingeniería, $8(1), 44-52$.

Henke, R.M., Benevent, R., Schulte, P., Rinehart, C., Crighton, K.A., \& Corcoran, M. (2016). The Effects of Telecommuting Intensity on Employee Health. American Journal of Health Promotion, 30(8), 604-612.

Hernández-Sampieri, R., Fernández-Collado, C., \& Baptista, P. (2014). Metodología de la investigación. México D.F.: McGraw-Hill.

Knox, E., Biddle, S., Esliger, D., Piggin, J., \& Sherar, L. (2014). Accounting for Sitting and Moving: An Analysis of Sedentary Behaviour in Mass Media Campaigns. Journal of Physical Activity \& Health, 12(9), 1198-1204.

Luque, P. M., \& Camargo, A. (2017). Teleworking and labor conditions. IUSLabor, 2, 5-18.

Martín, M., Barripedro, M., Martínez del Castillo, J., Jiménez-Beatty, J., \& Rivero-Herráiz, A. (2014). Diferencias de género en los hábitos de actividad física de la población adulta en la Comunidad de Madrid. Revista Internacional de las Ciencias del deporte, 10(38), 319-335.

Mikel, P. J., Ander, R. J., \& Urdampilleta, A. (2012). La prescripción de la actividad físico-deportiva según la edad. EFDeportes, Revista Digital, 16(165), 1-5. 
Núñez, A. N., \& Quirós-Ramírez, R. A. (2016). El teletrabajo en Costa Rica, un estudio exploratorio sobre su implementación en el Gran Área Metropolitana. Hacia la Sociedad de la Información y el Conocimiento en Costa Rica: Informe 2017. (295-332). San José, Costa Rica: PROSIC-UCR. Recuperado de http://www.prosic.ucr.ac.cr/sites/default/files/recursos/c6_dig2017.pdf

Ordóñez, P. A. (2018). Factors That Influence Job Satisfaction of Teleworkers: Evidence From Mexico. Global Journal of Business Research, 12(1), 41-49.

Organización Internacional de Trabajo. (2016). Las dificultades y oportunidades del teletrabajo para los trabajadores y empleadores en los sectores de servicios de tecnología de la información y las comunicaciones (TIC) y financieros. Recuperado de sector/documents/publication/wcms_531116.pdf

Pérez, S. C. (2010). El teletrabajo: ¿Más libertad o una nueva forma de esclavitud para los trabajadores?. Revista de Internet, Derecho y Política, (11), 22-33.

Sánchez, P. G., Montenegro, R. A., \& Medina, C. P. (2019). Teletrabajo una propuesta de innovación en productividad empresarial. Digital Publisher, 5(1), 91-104.

Secretaría de Salud Laboral y Secretaría de las Mujeres. (2017). El teletrabajo desde la perspectiva de género y salud ocupacional. Servicios a la ciudadanía, 1, 7-5.

Sierra, C. Y., Escobar, S. S., \& Melo, S. A. (2016). Trabajo en casa y calidad de vida: una aproximación conceptual. Cuadernos Hispanoamericanos de Psicología, 14(1), 57-72.

Tapasco, O., \& Giraldo, J. (2016). Factores Asociados a la Disposición por el Teletrabajo entre Docentes Universitarios. Ciencia y trabajo, 18(56), 87-93.

Tavares, A. I. (2017). Telework and health effects review. International Journal of Healthcare, 3(2), 30-36.

Universidad de Costa Rica. (2016). Encuesta actualidades 2016. Recuperado de: http://www.ucr.ac.cr/medios/documentos/2017/estadistica-encuesta-actualidades-2016-1-1.pdf

Vicente-Herrero, T., Torres, A. I., Torres, V. A., Ramírez, I. d., \& Capdevila, G. L. (2018). El Teletrabajo en salud laboral. CES Derecho, 9(2), 287-297.

Vries, H., Tummers, L., \& Vekkers, V. (2019). The Benefits of Teleworking in the Public Sector: Reality or Rhetoric. Review of Public Personnel Administration, 39(4), 570-593.

Walter, L. N., Pérez-Simon, C., Nagham-Ngwessitcheu, E., \& Vázquez-Ubago, M. (2014). Teletrabajo, un enfoque desde la perspectiva de la salud laboral . Medicina y seguridad del trabajo, 60(236), 587-599. 